\title{
Quantification of grain breakage during creep based on X-ray microtomography
}

\author{
Giuseppe Buscarnera ${ }^{1, *}$, Changbum Sohn $^{2}$, and Dawa $\mathrm{Seo}^{1}$ \\ ${ }^{1}$ Northwestern University, Department of Civil and Environmental Engineering, 60208 Evanston, United States \\ ${ }^{2}$ WSP USA, 63102 St Louis, United States
}

\begin{abstract}
Delayed compression is among the leading causes of long-term deterioration in granular systems, especially when it is mediated by the action of pore fluids. This time-dependent process is often classified as 'creep', a term conveying time-dependence without specifying the causes of deformation. This paper presents a methodology based on X-ray synchrotron microtomography to track delayed microstructural changes in compacted sand. Experiments on materials characterized by different grain size and shape have been designed to measure macroscopic variables such as strain rate, as well as to visualize the topological and morphological alterations of the constituting particles. The results reveal that nonnegligible inelastic processes such as grain breakage manifest during the first stages of loading, as well as during the ensuing constant-stress delayed compaction. A substantial role of the grain morphology was detected in both stages. Specifically, while samples made of angular grains displayed early breakage due to the exacerbated fragility of the particles, specimens made of rounded grains did not develop a markedly polydisperse structure prior to creep, which led to comparably more intense delayed fracturing. Furthermore, samples consisting of round grains were also found to exhibit more intense shape alterations, with morphological indicators that tended to converge over time towards those of initially angular grains. These results suggest that characterization and simulation of creep in granular media need to encompass a variety of microscopic processes controlled by grain-scale properties, thus requiring multi-scale testing and modelling techniques.
\end{abstract}

\section{Introduction}

The time-dependent behaviour of granular materials is crucial for the study of various phenomena, such as pile creep [1] and reservoir quality deterioration [2]. Recent studies indicate that particle rupture is among the key factors triggering delayed deformation in granular media [3]. For this reason, numerous recently developed constitutive models link macroscopic inelasticity to grain-scale processes, such as contact-scale strain energy storage and subcritical grain fracturing [4-6]. Most of these approaches have been built within the framework of breakage mechanics [7], a theory which captures the development of deformation in granular assemblies as the outcome of elastic energy release via grain crushing. Such formalism introduces a breakage state variable, $B$, which is directly linked with the microscopic fabric (e.g., the grain size distribution) [7]. In the context of creep, it has been recently shown that this theory can also be cast in a viscoplastic form by modelling the growth of breakage over time [5]. Such incorporation of subcritical crack growth under constant load led to a framework capturing time effects such as creep and relaxation, as well as time dependence due to the coupling between breakage and plastic strains (Figure 1). Such new class of constitutive laws requires characterization tools able to account for particle-scale processes, including microscale analyses able to track the evolution of grain size and morphology. In this context, Sohn and Buscarnera [8] tested the performance of rate-dependent breakage models through laboratory experiments and model simulations. The results showed that the particle size and shape affects the timescale of breakage, as well as that breakage and creep strain were accumulated simultaneously. However, the macroscopic nature of the tests implied a stepwise determination of breakage growth with multiple samples, thus not allowing the determination of the grain-scale energy release processes. Recent developments in the area of X-ray microtomography offer promising prospects to mitigate these shortcomings. For example, recent results obtained by Andò et al. [9] show that assemblies subjected to high stress slowly deform as a result of the propagation of fractures within a limited number of grains. Despite the promising nature of these results, the analysis was conducted only for part of the sample, which constrains the possibility of establishing a statistical connection between the evolution of the microstructure of the entire system and its continuum-scale manifestation in the form of delayed compaction.

To address this challenge, this paper aims to track the delayed grain fracture events taking place in a

\footnotetext{
* Corresponding author: g-buscarnera@northwestern.edu
} 


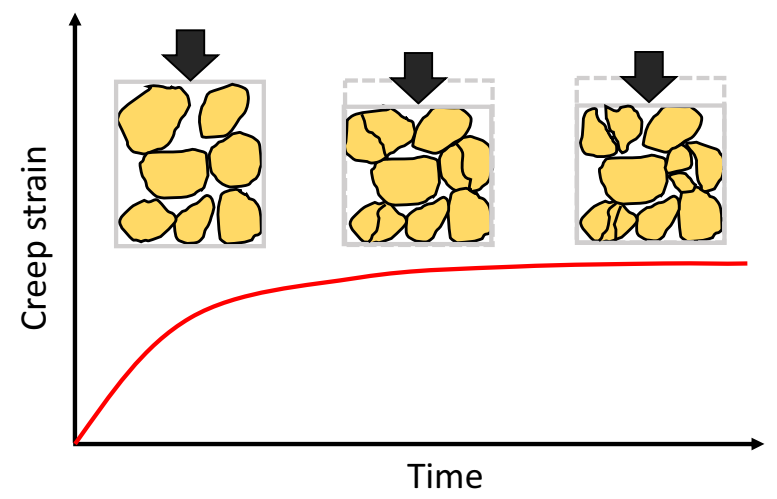

Fig. 1. Schematic of delayed breakage of granular materials.

granular assembly subjected to creep. In particular, to describe the role of the grain morphology, the analysis will be conducted with reference to materials characterized by different size and shape of their constituting particles. Specifically, these materials will be subjected to vertical stress able to engage the fracture strength of the particles and will subsequently subjected to in-situ creep tests performed in conjunction with 3D $\mathrm{X}$-ray tomography. The digital images resulting from these experiments will then be analysed through a particle tracking algorithm to inspect the microscopic mechanisms by which delayed breakage was developed.

\section{Experimental methods}

Two angular quartz sands with different particle size and a round quartz sand were used in the experiments (Table 1). Sphericity (S) was calculated according to the method proposed by Wadell [10]:

$$
S=\frac{\sqrt[3]{36 \pi V^{2}}}{A}
$$

where $V$ is the volume of an individual particle and $A$ is its surface area. The so-called flatness-elongation ratio (FE) was quantified as the ratio between longest length $\left(d_{L}\right)$ and shortest length $\left(d_{S}\right)$, as follows.

$$
F E=d_{L} / d_{S}
$$

Table 1. Material properties.

\begin{tabular}{|c|c|c|c|}
\hline Sand & D $50[\mathbf{m m}]$ & Sphericity & FE ratio \\
\hline Ottawa & 0.60 & 0.86 & 1.62 \\
\hline Q-ROK\#2 & 0.67 & 0.69 & 1.74 \\
\hline Q-ROK\#4 & 1.00 & 0.49 & 1.86 \\
\hline
\end{tabular}

The creep experiments were performed with a customdesigned miniaturized loading apparatus able to impose vertical stresses within the crushing regime of the tested materials [11]. The sands were oven dried at $110{ }^{\circ} \mathrm{C}$ for 24 hours prior to the test, dry-pluviated in an aluminum ring of 4.1-mm diameter and 5-mm height and densified by tamping. Such sample size, which was required to guarantee high-resolution scans, clearly constitutes a limitation in terms of the number of particles within the specimens. The samples were saturated with de-ionized water and open drainage boundaries. The vertical stress was applied under displacement-control at a rate of 0.05 $\mathrm{mm} / \mathrm{min}$, then switching to load-control during the creep stage. All sands were subjected to creep for a fixed duration of 4 hours. In addition, to compare the results obtained for different material systems, the samples were subjected to a normalized stress $\sigma / \sigma_{\mathrm{y}}=1.33$, where $\sigma_{\mathrm{y}}$ indicates the yield stress at the onset of breakage (Figure 2). The experiments were all conducted at the beamline 13-BM-D GeoSoilEnviro-CARS (GSECARS) of the Advanced Photon Source (APS) of the Argonne National Laboratory (Lemont, IL, USA), which allows for highresolution X-ray microtomography $(4.28 \mu \mathrm{m}$ per pixel). Digital images were collected through consecutive scans at 30-minutes intervals. Hereafter, selected scans will be discussed with reference to intact state, conditions prior to creep and conditions at the end of the tests.

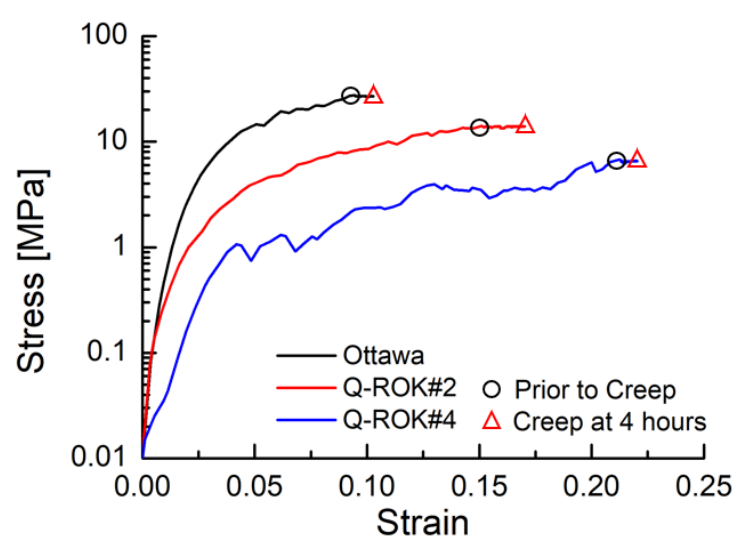

Fig. 2. Stress-strain curves measured for the three sands tested through the miniaturized loading apparatus.

\section{Image analysis}

To inspect grain crushing and quantify the evolution of the particle morphology during loading and creep stages, a tracking algorithm based on the sequential 3D X-ray tomography images was used [12]. The features of the algorithm, as well as the results derived from its use are outlined hereafter.

\subsection{Image processing}

Raw tomography images were pre-processed through the IDL software available at APS [13], and post-processed through the software Avizo. The images were filtered by anisotropic diffusion and solids and pores were separated with an iterative thresholding strategy, through which the particles were segmented and labelled individually. This procedure enabled the extraction of basic data regarding particle locations and morphology. 


\subsection{Tracking particles}

Quantifying how individual particles respond to creep is crucial to understand the macroscopic response emerging from the assembly. The challenge here is that the grains change their position and morphological properties from scan to scan, complicating the definition of kinematic quantities. To circumvent the problem, the algorithm used here matches grains which were labelled differently in the consequential scans by distinguishing two classes of units: intact and broken particles.

\subsubsection{Matching intact particles}

The algorithm verifies the characteristics of individual particles (i.e., potential position within 30 voxels of the designated window, the volume with a $\pm 3 \%$ tolerance, the length ratio with a $\pm 10 \%$ tolerance). When a particle is matched with a grain in another scan, those particles are considered as an identical units.

\subsubsection{Measurement of breakage evolution}

The particles which in the previous step are not assigned a match are treated as units which experienced a critical morphological variation between scans. Parent particles (intact particles in the previous scan) are thus matched with their child particles (newly generated fragments in the current scan) by adding child particles located in the observation window with an expected displacement until the cumulated fragment volume matches the volume of the parent grain within a given tolerance. Once parent and child grains are matched, the algorithm was used to inspect the modes of grain fracture. Specifically, in this work three modes were considered, namely splitting, chipping and comminution (Table 2).

Table 2. Definition of the three classes of hypothesized grain fracture mechanisms considered in this study.

\begin{tabular}{|c|c|c|c|}
\hline Sand & Splitting & Chipping & Comminution \\
\hline $\begin{array}{c}\text { Number of } \\
\text { fragments }\end{array}$ & $<4$ & - & $\geq 4$ \\
\hline $\begin{array}{c}\text { Volume of } \\
\text { Largest } \\
\text { fragment }\end{array}$ & $\leq 0.95$ Vol $_{\text {parent }}$ & $>0.95$ Vol $_{\text {parent }}$ & $\leq 0.95$ Vol parent \\
\hline
\end{tabular}

\section{Experimental results}

Preliminary compression tests were used to determine the yielding stress of the three sands (Ottawa sand - 20 MPa; Q-ROK\#2 - 10.50 MPa; Q-ROK\#4 - $5 \mathrm{MPa}$ ). The stress level for the creep stage was then chosen to ensure a constant stress ratio $\sigma / \sigma_{\mathrm{y}}$ of 1.33 in all materials. The stress-strain curves resulting from the tests are plotted in Figure 2. After 4 hours of creep, delayed deformation was observed in all sands.

Figure 3 shows the raw X-ray images of selected cross-sections relative to pre- and post-creep conditions for Ottawa, Q-ROK\#2 and Q-ROK\#4 sands. Figure 3a, $3 \mathrm{~b}$ and $3 \mathrm{c}$ show the breakage at $\sigma / \sigma_{\mathrm{y}}=1.33$ before creep. At this same stress ratio, most particles suffered crushing in Q-ROK\#4, while a much smaller proportion of Ottawa sand displaying fracturing under similar conditions. This result suggests that while stress concentration at irregular

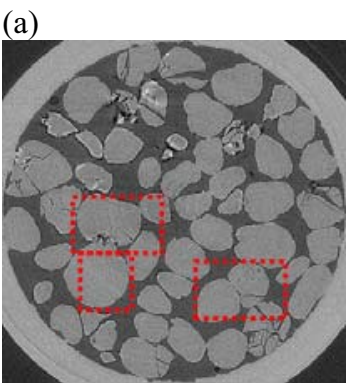

(b)

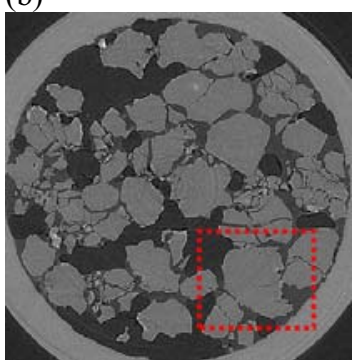

(c)

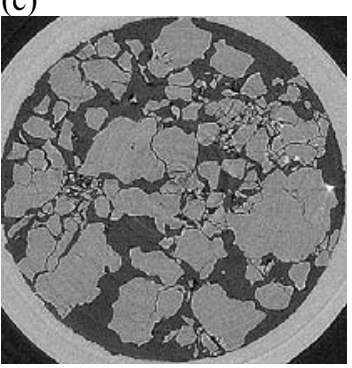

(d)

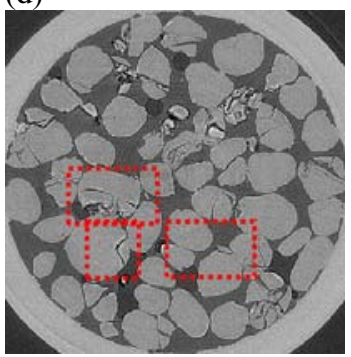

(e)

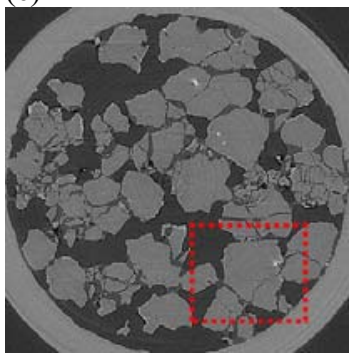

(f)

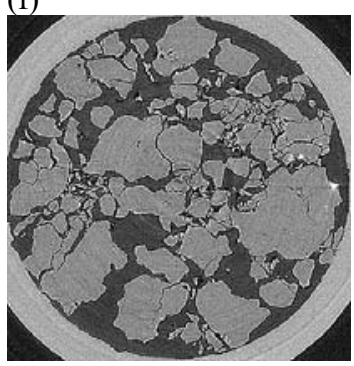

Fig. 3. Cross-sections of X-Ray scans of the tested sands; prior to creep (a) Ottawa (b) Q-ROK\#2 (c) Q-ROK\#4, 4 hours creep (d) Ottawa, (e) QROK\#2, (f) QROK\#4.

contacts makes angular sands more fragile (Q-ROK\#2), crushing mechanisms are less pervasive in round sands (Ottawa sand). In addition, a comparison between sands with similar morphology but different mean grain size indicates that materials with large particles (Q-ROK\#4) tend to have higher probability of developing breakage due to the accentuated fragility of large, brittle particles.

During the ensuing creep phase, the sands were subjected to constant stress for extended time, 4 hours in this study. During this stage intra-grain flaws were allowed to further grow if the local force network was able to promote sustained tensile actions. The scans collected at the end of the test allow the quantification of this process (Figure 3d, 3e, 3f). Both Ottawa and QROK\#2 sand were found to experience delayed crack growth (particles marked in red), while Q-ROK\#4 sand displayed negligible damage. This result is in contrast with the high crushability of Q-ROK\#4 grains pointed out by the prior compression stage, and it can be seen as a consequence of the small number of particles in the specimen. In fact, by the time of creep initiation most Q- 
ROK\#4 sand grains are heavily fractured, thus causing a highly polydisperse structure which enhances cushioning and hinders the propagation of intra-grain flaws.

\section{Quantification of breakage}

The evolution of the grain size distribution (GSD) is plotted based on the percentage of particle-mass versus the diameter of equivalent sphere through image analysis in Figure 4. After compression, the GSDs of Q-ROK\#4

(a)

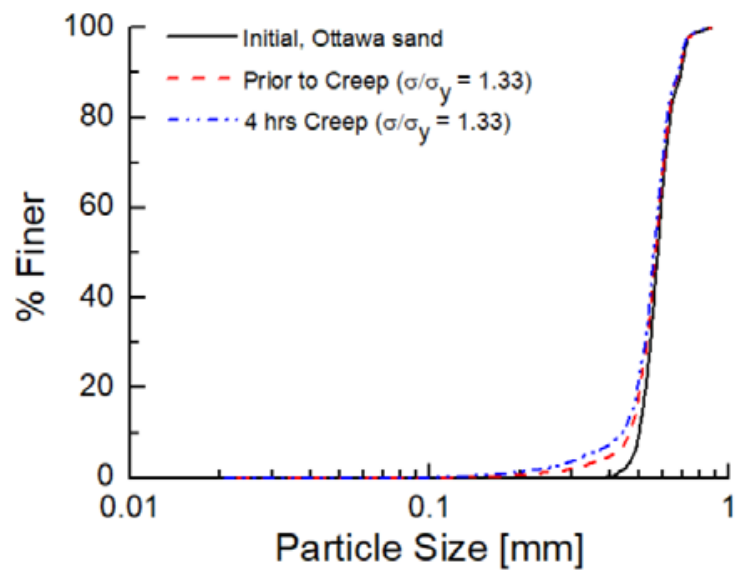

(b)

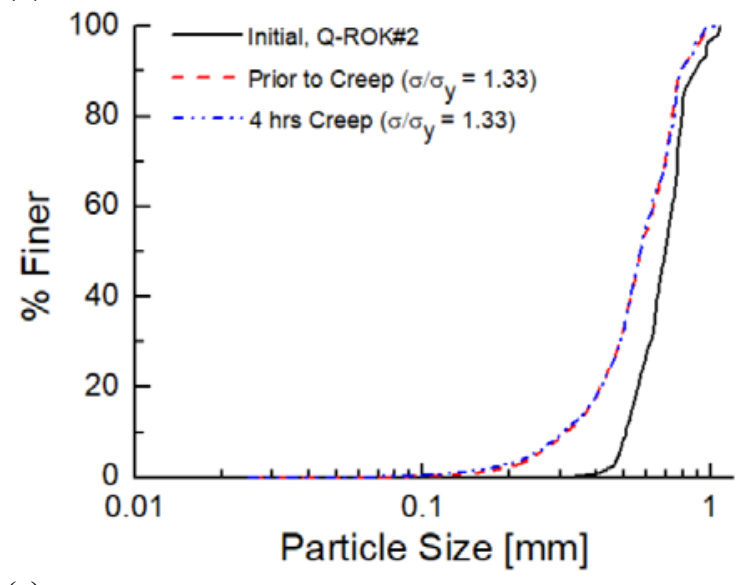

(c)

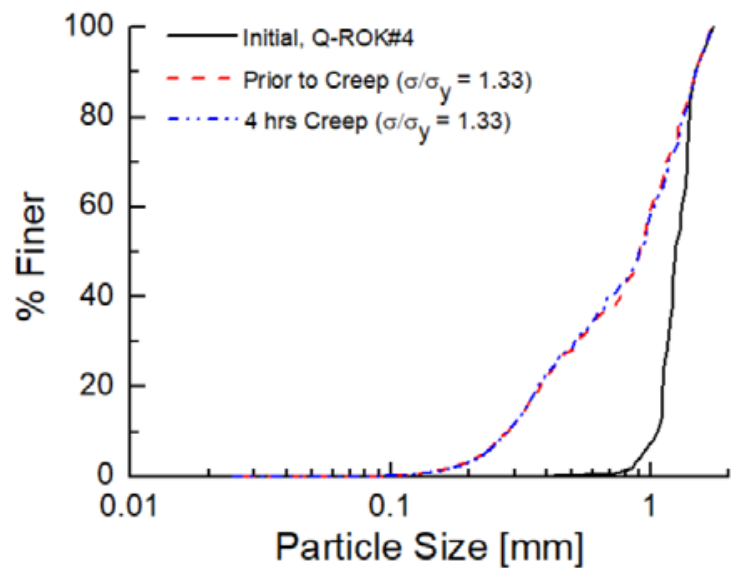

Fig. 4. Grain Size Distribution (a) Ottawa (b) Q-ROK\#2 (c) QROK\#4. and Q-ROK\#2 sands move significantly upwards, while the GSD of Ottawa sand displays limited changes. By contrast, during creep the GSD of Ottawa sand displays the largest variation, while the changes of Q-ROK\#2 and Q-ROK\#4 are negligible (Figure 4). Again, this result can be interpreted as an outcome of the different degrees of polydispersity of the three materials, with Ottawa being the most uniform sand, and thus the most likely to have low-coordination grains prone to delayed fracture.

To corroborate this point, Figure 5 displays the ratio of fragmented particles at three steps: initial condition, pre-creep stage and post-creep stage. This ratio reflects the percentage of broken particles over the intact particles. Upon loading, about $28 \%$ of round sand grains crushed while more than half of the angular sand grains crushed (nearly $74 \%$ and $95 \%$ for Q-ROK\#2 and QROK\#4, respectively). Upon creep, while the angular sands displayed slight breakage, the sand with round particles showed higher susceptibility to delayed fracture. Based on the total breakage events measured during creep, $95 \%$ of Q-ROK\#4 grains broke during compression, while $99 \%$ of the fragments fractured again during creep. In addition, Ottawa sand had creepinduced breakage in more than $89 \%$ of its fragments, thus displaying higher frequency of delayed grain-scale fracture in the broken particles.

Over time, subcritical cracks propagate within the grains and cause breakage across the sample. Figure 6 presents the evolution of two grain shape indicators, sphericity and FE ratio. Such average morphological properties were computed after volumetric thresholding (i.e., only for particles above a threshold volume of $10^{-}$ ${ }^{3} \mathrm{~mm}^{3}$ ), so to prevent a measurement bias affected by the numerous fine particles for which shape determination is uncertain. Prior to loading, Q-ROK\#4 displayed the most irregular shape ( 0.491 of sphericity and 1.856 of FE ratio) while Ottawa sand was characterized by the highest Sphericity (0.861) and lowest FE ratio (1.616). Such mismatch was gradually reduced upon compression and creep, with the morphology of all sands converging to similar values (Figure 6). Ottawa sand displayed the most intense shape variation by losing sphericity under both compression and creep. Although Q-ROK\#2 displayed a similar trend, its change of morphology was

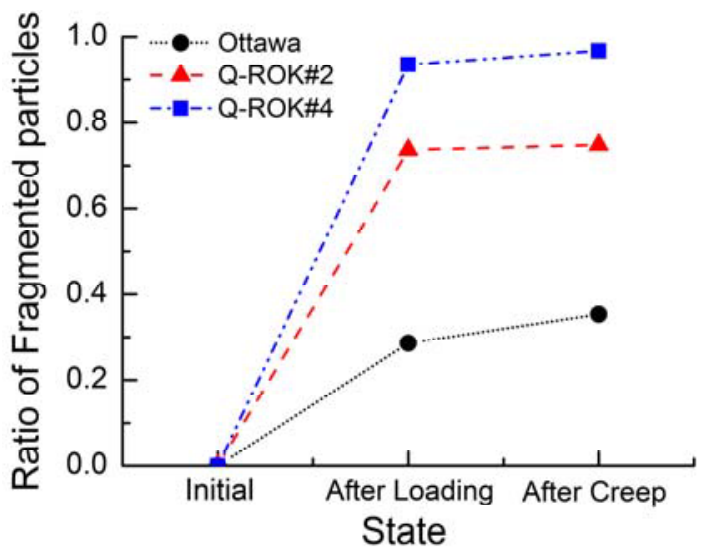

Fig. 5. Percentage of fragmented particles. 


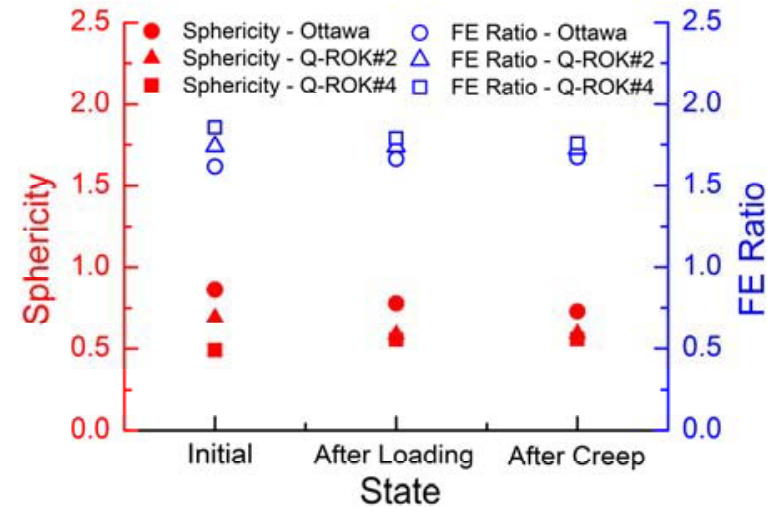

Fig. 6. Evolution of the average $3 \mathrm{D}$ shape parameters of the tested sands during loading and creep.

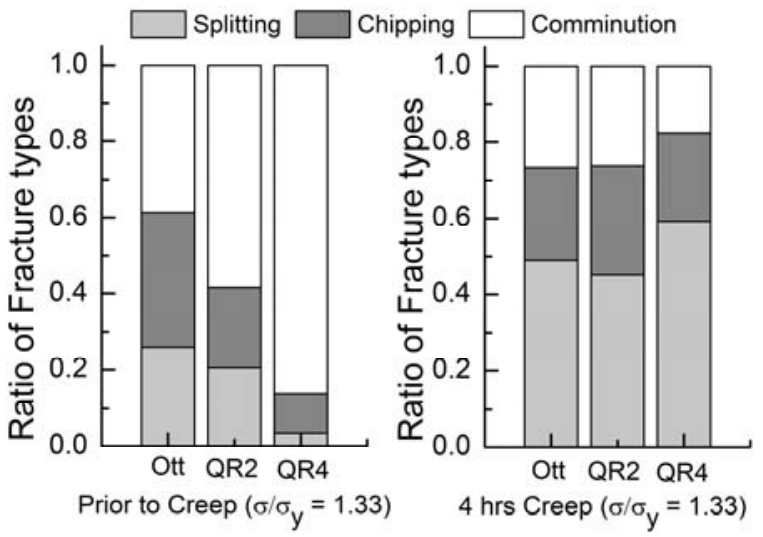

Fig. 7. Evolution of fracture modes of Ottawa sand, Q-ROK \#2 and Q-ROK \#4 in (a) Compression, (b) Creep.

weaker, especially during the creep stage. By contrast, the sphericity of Q-ROK\#4 was found to increased considerably in compression, and again rose slightly during creep. In terms of FE ratio, Q-ROK\#4 sand has an initial value $12 \%$ higher than that of Ottawa, corresponding to a more elongated shape. Such difference drops to less than 5\% upon creep with nearly identical final values of FE ratio for the two materials. Again, Q-ROK\#2 displayed intermediate trends between those of the two above mentioned end members. In other words, similar to sphericity all sands tend to approach the same value of shape indicator upon increasing stress and time. This tendency was observed regardless of the initial morphology and size of the tested materials.

As previously outlined, in addition to shape indicators, the breakage tracking algorithm allows the identification of the particles which led to the formation of crushed fragments at any given scan. These features enabled the classification of the grain fracture modes acting during the stages of loading and creep. At this reference, Figure 7 illustrates the evolution of three fracture modes, whose features have been conventionally defined in Table 2 for the purposes of this study. For the compression stage such fracture modes were identified by comparing the digital images available for the initial scan with those collected at the end of the compression stage (i.e., at $\sigma / \sigma_{y}=1.33$ ). For the creep stage, the same procedure was repeated by comparing the breakage patterns detected at the final scan (after $4 \mathrm{hr}$ creep) and the scan prior to the initiation of constant-stress delayed deformation. Upon compression (Figure 7a) the prevalent grain failure mode was comminution (i.e., the rupture of a particle through the formation of a large number of fragments). Among the three sands, QROK\#4 showed the highest frequency of comminution events, in that most grains were already heavily fractured after loading. This is likely due to the accumulation of successive ruptures between scans. Figure $7 \mathrm{~b}$ focuses instead on the creep stage, showing that half the fracture events were due to splitting, which in this case resulted the most frequent mode of failure. It is arguable that also in this case the comminution events were the outcome of successive ruptures. However, since during creep the particle scale forces are likely to be relatively stationary, an individual rupture event leading to a split is more likely to cause a relaxation of the forces acting on the surrounding particles, thus reducing the likelihood of successive ruptures of the same grain. A refinement of these data can benefit from more frequent scans during both loading and creep, but it is invariably going to be constrained by the finite frequency at which the materials can be imaged.

\section{Conclusions}

This study provided a quantitative microscale analysis of delayed particle crushing during creep. Such process was studied by taking into account the role of the particle size and their morphological characteristics. For this purpose, three materials were used (i.e., round Ottawa sand with a $\mathrm{D}_{50}$ of $0.60 \mathrm{~mm}$, ant two angular sands - Q-ROK\#2 with a $\mathrm{D}_{50}$ of $0.67 \mathrm{~mm}$ and Q-ROK\#4 with a $\mathrm{D}_{50}$ of $1.00 \mathrm{~mm}$ ). One-dimensional compression creep experiments were conducted in combination with 3D X-ray tomography. In addition, a breakage tracking algorithm was used to classify the fracture modes emerging upon loading and creep (i.e., splitting, chipping or comminution). Upon compression, the morphological properties of the grains were found to affect the breakage of the sample, with coarse angular sands displaying accentuated crushability compared to round, finer counterparts. Such relation was inverted during creep, in that the major crushing suffered by angular sands during the loading stage likely created cushioning effects able to prevent delayed breakage. By contrast, round sands which did not benefit from such broadening of the grain size distribution were more susceptible to crushing over time at constant stress. In addition to macroscale variables, the algorithm allowed the tracking of average morphological indicators such as Sphericity and FE ratio. It was found that, despite the initial differences, the value of these shape indicators tended to converge towards similar values for all sands. This indicates that progressive breakage minimizes the original differences in grain morphology. In addition, the dominant modes of grain fracture during the different stages of the experiments were tracked. It was found that the splitting was the dominant grain failure mechanism during creep, as well as that most delayed fractures occur 
in already broken particles or fragments. The results provided in this work illustrate that the morphology and size of the particles of a granular material affect creep in a number of ways, either by impacting the rate at which individual grains break or by affecting the microscopic fabric of the material (and the ensuing cushioning effect) at the onset of constant-stress deformation. Quantifying these feedbacks and implementing them into mechanical models is therefore essential for any predictive approach aimed at assessing the performance of geotechnical and geophysical systems in light of the inherent constitution of its grain-scale building blocks.

This work was primarily funded by the U.S. Department of Energy (grant No. DE-SC0017615). Partial support from the U.S. Army Research Office (grant W911NF-19-1-0245) is also gratefully acknowledged. To conduct this research resources of the Advanced Photon Source, a U.S. Department of Energy (DOE) Office of Science User Facility operated by the Argonne National Laboratory under Contract No. DE-AC02$06 \mathrm{CH} 11357$ were used. In particular, the activities were carried out by benefiting of facilities at GeoSoilEnviroCARS (Sector 13-BM-D), which is supported by the National Science Foundation - Earth Sciences (EAR-1128799) and the Department of Energy, Geosciences (DE-FG02-94ER14466).

\section{References}

1. C.F. Leung, J.K. Lim, R.F. Shen, Y.K. Chow, J. Behavior of pile groups subject to excavationinduced soil movement. Geotech. Geoenviron., 129, 58-65 (2003)

2. M. Hettema, E. Papamichos, P.M.T.M. Schutjens, Subsidence delay: field observations and analysis. Oil. Gas. Sci. Technol., 57, 443-458 (2002)

3. Z. Wang, R.L. Michalowski, Contact fatigue in silica sand-Observations and modeling. Geomech. Energy. Envir, 4, 88-99 (2015)

4. Y. D. Zhang, G. Buscarnera, I. Einav. Grain size dependence of yielding in granular soils interpreted using fracture mechanics, breakage mechanics and Weibull statistics. Géotechnique, 66, 149-160 (2016)

5. Y.D. Zhang, G. Buscarnera, A rate-dependent breakage model based on the kinetics of crack growth at the grain scale. Géotechnique, 67, 953967 (2017)

6. Y.D. Zhang, G. Buscarnera, Breakage mechanics for granular materials in surface-reactive environments. J. Mech. Phys. Solids. 112, 89-108 (2018)

7. I. Einav, Breakage mechanics - part I: theory. J. Mech. Phys. Solids, 55, 1274-1297 (2007)

8. C. Sohn, G. Buscarnera, Measurement and simulation of comminution rate in granular materials subjected to creep tests. Granul Matter, 21, 60 (2019)

9. E. Andò, J. Dijkstra, E. Roubin, C. Dano, E. Boller, A peek into the origin of creep in sand. Granul Matter, 21, 11 (2019)
10. H. Wadell, Volume, shape, and roundness of quartz particles. J. Geol., 43, 250-280 (1935)

11. M. B. Cil, J. Schabelski, A. I. Packman, G. Buscarnera, A miniaturized testing apparatus to study the chemo-mechanics of porous media. Geotech. Test. J., 43 (2020)

12. D. Seo, C. Sohn, M. Cil, G. Buscarnera, Evolution of particle morphology and mode of fracture during the oedometric compression of sand. To appear in Géotechnique. (2020)

13. M. L. Rivers, TomoRecon: High-speed tomography reconstruction on workstations using multithreading). International Society for Optics and Photonics, 8506, 85060U (2012) 